Conclusion. 1. This quality improvement initiative has highlighted that the current level of information in referring patient to radiology is variable and dependent on the referrer.

2. All referrals should state exclusion criteria as per the NICE guidelines on neuroimaging in diagnosis of dementia.

3 . Preliminary evidence suggests that requesting specific radiological rating scales could improve the quality of information received in the imaging report. The second part of this quality improvement initiative will aim to explore the impact of requesting these scales routinely.

A prescription of information - promoting symptom self-management in people with functional neurological disorder (FND)

Hashim Dadah ${ }^{1 \star}$, Uzoma Anthony-Uzoeto ${ }^{1}$, Sadat Yazdouni ${ }^{1}$, Ali Aweis-Asanga ${ }^{1}$, Alan Dunlop ${ }^{2}$ and Rafey Faruqui ${ }^{3}$

${ }^{1}$ King's College London; ${ }^{2}$ Kent and Medway NHS and Social Care Partnership Trust and ${ }^{3}$ Kent and Medway NHS and Social Care Partnership Trust, University of Kent

${ }^{\star}$ Corresponding author.

doi: 10.1192/bjo.2021.493

Aims. Functional Neurological Disorder (FND) is known to be associated with high healthcare resource utilisation and poor quality of life. Patients' understanding of the disorder is considered instrumental in improving prognosis.

We produced a symptom self-management patient education strategy with a booklet and FND symptoms recording template in a community neuropsychiatry setting. We embedded this psychoeducation intervention in a post-nursing triage model of care. Method. A co-production cycle of patient education material was implemented as part of a Quality Improvement Project (QIP) at East Kent Neuropsychiatry Service. Year 4 medical students completed their first QIP cycle involving 4 students, 2 multidisciplinary team members and 4 patients with functional neurological presentations. An FND leaflet and symptom recording template was produced and reviewed using feedback domains such as leaflet readability, perceived usefulness, and template design. The revised version of leaflet was then pilot-tested in second QIP cycle via email or post to 12 patients awaiting their group psychology or neuropsychiatry appointments for treatment of FND. The uptake and impact of leaflet was assessed using telephonebased structured feedback collection.

Result. The first QIP cycle included 10 participants and generated qualitative knowledge domains, providing examples of different types of FND presentations and a biological-psychological-social model explaining onset and/or recurrence of FND symptoms. Group patient feedback and co-production input allowed inclusion of the patient voice and a re-design of leaflet and symptom recording template.

The second QIP cycle involved 12 participants: feedback was collected two weeks after circulation of patient education material. Only 5 participants (42\%) had read and used their education leaflet and template during this period. Patients described the booklet as useful overall, but thought it to be more useful at the point of diagnosis and referral to neuropsychiatry. Qualitatively, patients wished there to be more emphasis on FND being explained as "less psychiatric, more a neuropsychiatric problem", and that it would be "very good for someone who had just been diagnosed". $80 \%$ of responders rated the leaflet quality $8 / 10$ or above. These respondents felt that the leaflet had helped them understand their condition better than they did previously. Usefulness of an additional self-formulation flowchart was rated as $8 / 10$ or below by all patients - with several finding it difficult to use.
Conclusion. Our QIP supports the need for early patient education when discussing diagnosis of FND. The finding of $42 \%$ uptake within two weeks of leaflet dispatch is encouraging.

\section{Audit on High Dose Antipsychotic Treatment (HDAT) Monitoring at Rampton Hospital}

Astha Das* and Ian Yanson

Nottinghamshie Healthcare NHS Trust

${ }^{*}$ Corresponding author.

doi: 10.1192/bjo.2021.494

Aims.

- High Dose Antipsychotic Treatment defined as $100 \%$ of the maximum recommended dose in British National Formulary, either as single agent or in combination.

- HDAT and poly-pharmacy may be linked to heightened mortality for psychiatric patients. The Committee on Safety of Medicines, Medicines and Healthcare Products Regulatory Agency recommended ECGs, electrolyte monitoring after each dose escalation, and 6 monthly intervals.

- The Royal College of Psychiatrists in 2006 suggested some justifiable cases of temporary poly-pharmacy with careful monitoring.

- This audit has been done in past to improve standards, especially in High Secure Setting where prescribing HDAT is a common practice

- To audit adherence to "HDAT monitoring guidelines" including regular monitoring of bloods, physical observations and ECG, done after every dose escalation plus at every 6 months.

- To monitor compliance with consent to treatment documentation including reasons of being on HDAT, documentation of physical health monitoring results

\section{Method.}

- All patients prescribed high dose antipsychotic (regular and as required) were identified by treating Consultants and also going through drug cards.

- One year retrospective review of haematological, ECG and physical observations were identified through Electronic notes

\section{Result.}

- $6 \%$ of patients received HDAT within Rampton Hospital in 2018(12 males' vs 6 females).

- All patients on Regular HDAT had yearly TFT done whereas only $71 \%$ had prolactin monitoring done.

- Approximately $50-60 \%$ of patients had quarterly blood monitoring including glucose, electrolytes, lipids, liver function test and full blood count.

- About $40 \%$ of patients had quarterly ECG monitoring recorded.

- $100 \%$ patients on regular HDAT had quarterly physical observation monitoring compared to $81 \%$ patients on HDAT (including PRN).

- Consent forms were completed for all patients on HDAT. 85\% patients on regular HDAT has the reasons for treatment documented in the notes compared to $100 \%$ patients on HDAT (including PRN).

\section{Conclusion.}

- Improvement in monitoring of blood parameters and cardiac function (ECG) $40-60 \%$ as compared to 2014 audit (8\% to $23 \%$ ).

- Yearly monitoring of TFTs and Prolactin also appeared better (100\% and $71 \%$ ) which was (88\% and $72 \%$ in 2014$)$.

- Quarterly physical observations were recorded in $77 \%$ patients on regular HDAT in 2014 which improved to $100 \%$ in 2018. 